\title{
Pathological features of persistent adnexal masses in pregnancy
}

\author{
Zhiqiang Zhang ${ }^{1 \#}$, Xiaojuan Zheng ${ }^{2 \#}$, Miao Zhang ${ }^{3}$, Jinfeng Li $^{1}$, Jing Zhao ${ }^{1}$, Jing Zheng ${ }^{1}$, Shuzhen Wang ${ }^{1}$ \\ ${ }^{1}$ Department of Obstetrics and Gynecology, Beijing Chao-Yang Hospital, Capital Medical University, Beijing, China; ${ }^{2}$ Department of Obstetrics and \\ Gynecology, Fangshan District, Beijing Maternal and Child Health Hospital, Beijing, China; ${ }^{3}$ Department of Ultrasound Medicine, Beijing Chao- \\ Yang Hospital, Capital Medical University, Beijing, China \\ Contributions: (I) Conception and design: S Wang, Z Zhang; (II) Administrative support: S Wang; (III) Provision of study materials or patients: Z \\ Zhang, M Zhang, J Li; (IV) Collection and assembly of data: X Zheng, J Zhao, J Zheng; (V) Data analysis and interpretation: X Zheng, J Li, J Zhao; (VI) \\ Manuscript writing: All authors; (VII) Final approval of manuscript: All authors. \\ \#These authors contributed equally to this work. \\ Correspondence to: Shuzhen Wang. Department of Obstetrics and Gynecology, Beijing Chao-Yang Hospital, Capital Medical University, No. 8 \\ Gongren Tiyuchang Nanlu, Chaoyang District, Beijing, China. Email: darrywang2003@163.com.
}

Background: The composition of pelvic masses during pregnancy is complex, and over $>70 \%$ of masses are
physiology. Determining the source and nature of tumor tissue is incredibly important in clinical practice, as
the proportion of metastatic and low-grade tumors in adnexal masses increase during pregnancy. This study
sought to analyze the pathological features of persistent adnexal masses in pregnancy.

Methods: A retrospective case analysis method was adopted to summarize the pathologic features of persistent adnexal masses in pregnancy in 228 patients at the Beijing Chao-Yang Hospital, Capital Medical University.

Results: Mature teratoma was the most common histopathological type of persistent adnexal masses in pregnancy $(66 / 228,28.95 \%)$, followed by the corpus luteum of pregnancy $(38 / 228,16.67 \%)$. Borderline/ malignant tumors accounted for $5.70 \%(13 / 228)$ of tumors. Malignant adnexal tumor tissues were derived from multiple types of tissues. a single-factor analysis showed that borderline/malignant tumors had a significant relationship with the morphological features of mass in the ultrasound $(\mathrm{P}=0.000)$, and had a significant relationship with initial mass size $(\mathrm{P}=0.018)$. A multivariate binary logistic regression analysis indicated no certain independent risk factor. A significant difference in tumor pathology distribution was noted between those who underwent emergency surgery during pregnancy, elective surgery during pregnancy, and simultaneous tumor removal during cesarean section $\left(\chi^{2}=24.22, \mathrm{P}=0.000\right)$.

Conclusions: A special particularity exists in the pathology distribution of persistent adnexal masses in pregnancy. Additionally, extensive histological sources and the need to rule out borderline/malignancy for solid cystic tumors were noted.

Keywords: Pregnancy; adnexal mass; clinical feature; pathological feature; surgery

Submitted Apr 02, 2021. Accepted for publication May 28, 2021.

doi: $10.21037 / \mathrm{atm}-21-2137$

View this article at: http://dx.doi.org/10.21037/atm-21-2137

\section{Introduction}

Adnexal masses are not uncommon in pregnancy. The majority of such masses represent physiological changes that are spontaneously relieved throughout pregnancy. $<1 / 3$ of these mases persist throughout pregnancy and require surgical treatment during or after pregnancy
(1-3). Determining the pathological features of persistent adnexal masses in pregnancy is very important in deciding which management approach to adopt. Treatment is also decided based on the nature of a tumor. The effect of a mass on pregnancy should be taken into account to avoid unnecessary surgery and delays in treatment, which can cause delays in diagnosis that interfere with or can endanger 
the mother and fetus. During clinical practice, the collective intelligence of doctors from different departments is required to undertake a comprehensive individualized analysis based on the characteristics of the mass, the risk of potential complications, the tumor type, gestational age, and the family's desire for the fetus. This study analyzed the pathological features of persistent adnexal masses in pregnancy in 228 patients at Beijing Chao-Yang Hospital, Capital Medical University to provide a basis for the reasonable clinical management of adnexal masses in pregnancy.

We present the following article in accordance with the STROBE reporting checklist (available at http://dx.doi. org/10.21037/atm-21-2137).

\section{Methods}

\section{Study subjects}

This study was approved verified by the Hospital's Institutional Review Board, and 271 patients from the Department of Obstetrics at Beijing Chao-Yang Hospital, Capital Medical University, between January 1, 2012, and December 31, 2019, with confirmed diagnoses of persistent adnexal masses during pregnancy were enrolled in the study. The management strategies adopted for adnexal masses may differ during pregnancy and puerperium (puerperium means from given her baby to the 42 days). In this study, 43 patients, who still had masses 42 days after giving birth or had been lost to follow-up, were excluded. Demographics and medical records from intrauterine pregnancy diagnosis to 1 year postpartum were collected for each patient from the electronic medical record system for the remaining 228 cases of persistent adnexal masses in pregnancy. The data collected included age, pregnancy, birth, tumor family history, and medical history. Pregnancy medical data (early, middle, and late pregnancy ultrasound images and reports) and pregnancy outcomes were also collected. The patients were aged 19-46 years old. The end of follow-up was set at 1 year postpartum. However, telephone or outpatient follow-ups were carried out if a patient's electronic medical records were unclear.

To be eligible to participate in the study, patients had to meet the following inclusion criteria: (I) have an adnexal mass found by examination before or during early pregnancy and/or an ultrasound-diagnosed adnexal mass $\geq 3 \mathrm{~cm}$ (dynamic observation had to show that the mass was persistent through 16 weeks of pregnancy); (II) have a clinically or imaging-diagnosed adnexal mass during middle or late pregnancy; and (III) have an undiagnosed adnexal mass discovered during cesarean section. Patients were excluded from the study if they met any of the following exclusion criteria: (I) had an adnexal mass diagnosed during early pregnancy, and disappearance latter during pregnancy; (II) had a medical history of a malignant tumor in another system; (III) had a persistent adnexal mass but rejected surgery; (IV) had incomplete clinical data; and/or (V) had a mass that was still present at day 42 of the postpartum follow-up period.

\section{Ultrasound diagnostic standard for an adnexal mass in pregnancy}

Based on the physical features of an ultrasound, an adnexal mass may be classified as cystic, cystic solid, or solid (4).

\section{Cystic mass characteristics}

(I) Under an ultrasound, if the ovary shows an anechoic fluid sonolucent area with clear margins, accompanied by an enhanced posterior wall and posterior echo with a thin wall, and mild blood flow in the capsule, it will usually be a functional cyst, which may be a follicular cyst, corpus luteum, theca lutein cyst, polycystic ovary, or ovarian hematoma. (II) The characteristics of a mesonephric or paramesonephric cyst include a single compartment, thin walls, a circular mass with an anechoic fluid sonolucent area located within the mesosalpinx between the ovarian hilum and fallopian tube. (III) An adnexal neoplastic cyst will most likely be a serous cystadenoma if it is unilateral, and has a single compartment, a smooth and thin cystic wall, a circular or oval anechoic area with clear boundaries, and an enhanced posterior wall and echo. A unilateral and multicompartment cyst with thin and weak diffused hyperechoic spots and hyperechoic septa with multiple different-sized compartments will most likely be a mucinous cystadenoma or a dermoid cyst.

\section{Cystic solid mass characteristics}

A cystic mass with thick walls, a protruding interior wall, blood-flow signals, and rich vascularity in the solid area will most likely be an epithelial cystadenoma or a cystadenocarcinoma. A structure with heterogeneous echoes and bone structure accompanied by a fat-fluid level will 
most likely be a teratoma.

\section{Solid mass}

A homogenous solid mass with clear boundaries and bloodflow signals will most likely be a sex cord-stromal tumor. An irregular hyperechoic solid mass may be a teratoma.

\section{Malignant tumor characteristics}

A malignant tumor is solid or cystic solid, has a protruding interior wall, rich vascularity, low resistance index (RI) value and complete or incomplete capsulation with or without ascites.

\section{Surgical timing and indication of an adnexal mass in pregnancy}

\section{Indication for emergency surgery}

Emergency surgery is required if (I) there is a diagnosis of a pelvic mass in pregnancy with a suspected mass pedicle torsion, and (II) a suspected ruptured adnexal mass is confirmed.

\section{Indication for elective surgery during pregnancy}

Elective surgery is required if (I) observation shows an adnexal mass is persistent over 16 weeks of pregnancy, a physiologic cyst has been excluded and surgery is requested by the patient (II) a malignant or borderline tumor is suspected (III) there is an increase in mass size during pregnancy (i.e., the mass reaches $>10 \mathrm{~cm}$ in diameter), or (IV) an adnexal mass is found during the cesarean section or by another method at full- or near full-term pregnancy.

\section{Statistical method}

Statistical Package for the Social Sciences, version 22.0, software was used. Count data are represented by frequency (percentage), and the $\chi^{2}$ test was used for intergroup comparisons. Quantitative data are represented as mean \pm standard deviation, and an analysis of variance was conducted for intergroup comparisons. A multiple logistic regression analysis was undertaken to analyze the effects of the pathologic features. A $\mathrm{P}<0.05$ was used to determine statistical significance.

The clinical study was approved by the Ethic Committee of Beijing Chao-Yang Hospital (No. 2021-science-210) and was conducted in accordance with the provisions of the Declaration of Helsinki (as revised in 2013). Individual consent for this retrospective analysis was waived.

\section{Results}

\section{Pathological features of a persistent adnexal mass in pregnancy}

Of the 228 patients enrolled in this study, 68 (29.821\%), 54 (23.7\%), 2 (0.9\%), 4 (1.8\%), 34 (14.9\%), 28 (12.3\%), and $38(16.7 \%)$ patients had germ cell tumors (66 mature teratomas, and 2 immature teratomas), epithelial tumors (34 serous tumors, and 20 mucous tumors), sex cord-stromal tumors, metastatic malignant tumors, endometriosis, ovarian tumor-like cysts, and corpus luteum, respectively.

\section{Relationship between the morphological features of a persistent adnexal mass in pregnancy and histopathology}

The morphological features of a persistent adnexal mass in pregnancy can be categorized into cystic, cystic solid, and solid based on an ultrasound. Of the 228 patients, 150 (65.8\%), $34(14.9 \%)$, and $44(19.3 \%)$ patients had cysts, cystic solid masses, and solid masses, respectively. Of the 150 patients with cystic masses, 2 patients $(1.3 \%)$ had borderline tumors, and no patients had malignant tumors. Of the 44 and 34 patients with solid and cystic solid masses, 8 $(10.4 \%)$ and 3 (3.9\%) had malignant and borderline tumors, respectively. A Fisher's exact test indicated a significant difference $(\mathrm{P}=0.000)$ in pathology type distribution between tumors with different morphological features (see Table 1).

\section{Relationship between the initial size of a persistent} adnexal mass in pregnancy and the pathology results Based on the largest diameter of $6 \mathrm{~cm}$, the physiologic adnexal masses in pregnancy were divided into 2 groups $(<6$ and $\geq 6 \mathrm{~cm}$ groups). Patients with $<6$ and $\geq 6 \mathrm{~cm}$ diameter masses may have had physiologic cysts and pathologic tumors, respectively (5). The masses were collectively categorized into the 4 following types: a benign teratoma, a benign epithelial tumor, a borderline/malignant tumor, or an ovarian tumor-like cyst (endometriosis cyst, paraovarian cyst, simple cyst, and corpus luteum; see Table 1). A significant difference was found in the pathological distribution of tumors with different initial sizes $\left(\chi^{2}=8.06, \mathrm{P}=0.034\right)$.

\section{Growth of a persistent adnexal mass in pregnancy}

The ovarian masses were routinely monitored throughout pregnancy from the first examination in early pregnancy to the end of pregnancy. During the patient follow-up period, the diameter of each mass was measured. Based on 
Table 1 Relationship between the morphological features of a persistent adnexal mass in pregnancy and the surgical method and histopathology

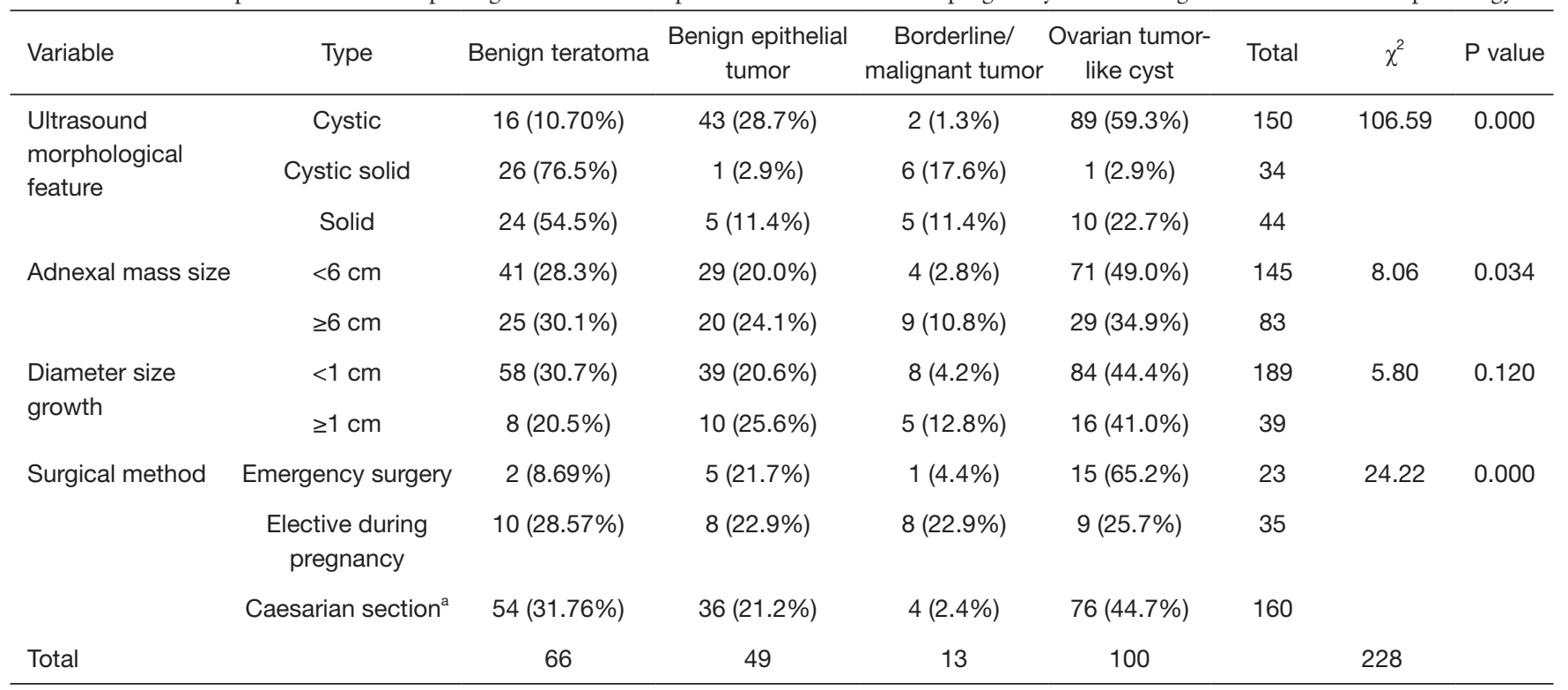

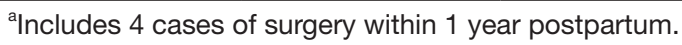

the differential diagnoses of benign or malignant tumors, a $1-\mathrm{cm}$ increase in a mass (as observed in the ultrasound) was considered tumor growth (6). Table 1 shows the relationship between tumor growth during pregnancy and pathological type. Notably, the $\chi^{2}$ test indicated that an increase in the largest tumor diameter was not related to the pathological results $\left(\chi^{2}=5.80, \mathrm{P}=0.120\right)$.

Effects of surgery timing on the pathological results of persistent adnexal masses in pregnancy

Of the 228 patients, 58 cases under went surgery during pregnancy (of whom, 23 underwent emergency surgery and 35 underwent elective surgery), 166 cases received no intervention during pregnancy, underwent a resection during the cesarean section, and 4 cases underwent surgery within 1 year postpartum.

Emergency surgery during pregnancy was performed to treat 22 cases of mass pedicle torsion and 1 case of a suspected ruptured mass. Of the 35 cases of elective surgery, $8,8,10$, and 9 were suspected to be borderline/malignant tumors, benign ovarian epithelial tumors, benign teratomas, and tumor-like cysts, respectively. Of those who underwent mass resection during cesarean section, 2 cases had malignant tumors (1 granulosa cell tumor and 1 immature teratoma) and 2 cases had borderline noninvasive epithelial tumors. A Fisher's exact test indicated no difference in the distribution of pathology types for the adnexal masses resected by emergency surgery, elective surgery, or during cesarean section $(\mathrm{P}=0.183$; see Table 1$)$.

\section{Borderline/malignant tumor risk assessment for a persistent adnexal mass in pregnancy}

Of the 228 cases, 5, 8, and 215 cases had borderline tumors, malignant tumors, and benign tumors or tumor-like cysts, respectively. A comparison was made of the patient age distributions for those with malignant/borderline tumors (19-39 years old; median age, 30 years old; mean age, $30.85 \pm 5.97$ years old) and those with benign tumor/tumorlike cysts (21-46 years old, median age, 31 years old; mean age, $30.86 \pm 4.018$ years old). No significant difference was found between the 2 patient groups $(\mathrm{t}=0.008, \mathrm{P}=0.994$; see Table 1). Additionally, a single-factor analysis was undertaken to identify influential factors in borderline/ malignant tumors, and the results showed that borderline/ malignant tumors had no relationship with gestational age at diagnosis $\left(\chi^{2}=0.117, \mathrm{P}=0.751\right)$, had a significant relationship with the morphological features of mass in the ultrasound $(\mathrm{P}=0.000)$, had no relationship with mass growth during pregnancy $(\mathrm{P}=0.051)$, and had a significant relationship with initial mass size $(\mathrm{P}=0.018$; see Table 2$)$. A multivariate binary logistic regression analysis indicated no 
Table 2 Single-factor analysis of influential factors of borderline/malignant tumors

\begin{tabular}{|c|c|c|c|c|}
\hline Types & Malignant and borderline tumor & Benign tumor and tumor-like cyst & $\mathrm{t} / \chi^{2}$ & $P$ value \\
\hline Gestation age at diagnosis & $17.15 \pm 14.74$ & $14.69 \pm 13.62$ & 0.117 & 0.751 \\
\hline $\begin{array}{l}\text { Ultrasound morphological } \\
\text { features }\end{array}$ & & & 17.55 & 0.000 \\
\hline Cystic solid & 7 & 39 & & \\
\hline Initial mass size & & & 6.22 & 0.018 \\
\hline$<6 \mathrm{~cm}$ & 4 & 140 & & \\
\hline
\end{tabular}

certain independent risk factor.

\section{Discussion}

\section{Pathological features of adnexal masses in pregnancy}

This study conducted a retrospective analysis of 228 patients with adnexal masses during pregnancy at Beijing Chao-Yang Hospital, Capital Medical University. The results showed that the pathological distribution and tissue source of masses during pregnancy were particularly different from those during non-pregnancy (7). With a composition ratio of nearly $30 \%$, germ cell tumors were the most common tissue source for adnexal masses in pregnancy (a figure lower than that reported in other literature), followed by corpus luteum at $16.7 \%$. Similar to other studies, we concluded that cystic teratoma was the most common tumor in pregnancy (40-50\%); however, the proportion of corpus luteum and simple cysts is also very high (11-41\%) (1-3). Additionally, we concluded that the tissue sources of the malignant tumors were multiple. Of the 228 patients enrolled in this study, 9, 1, 2, 1, and 1 had malignant tumors ( 3 metastatic malignant tumors originated from the intestinal tract), non-Hodgkin lymphoma, immature teratomas, granulosa cell tumors, and ovarian epithelial malignant tumors, respectively. The malignant tumor proportion was $3.51 \%$; this figure is similar to that reported in other literature $(8,9)$. The proportion of ovarian borderline/malignant tumors in pregnancy was different from that in non-pregnancy (10).

\section{Difficulties in clinically diagnosing persistent adnexal masses in pregnancy}

Due to developments in related disciplines and complete perinatal health care, adnexal masses that have no chance of being diagnosed before pregnancy can be diagnosed in pregnancy. Consequently, the diagnosis rate of ovarian masses in pregnancy has increased. However, this has also resulted in a certain degree of over diagnosis. During pregnancy, the physiologic existence of the corpus luteum along with an increase in estrogen and progesterone levels increases the incident rate of physiologic cysts and also results in a certain false-positive rate. Additionally, an enlarged pregnant uterus makes diagnosis difficult. Increased false-positive and false-negative rates can lead to problems of over- and under-treatment. There is no better diagnostic method than ultrasound currently exists.

Radiation contact should be avoided during pregnancy, as the ability of computed tomography (CT) to distinguish soft tissue was contraindicated. Thus, the application of CT in pregnancy is limited. Magnetic resonance imaging (MRI) can be used to scan the pelvic cavity during pregnancy. The American College of Radiology has noted that there is no evidence of fetal development influence from MRI examination during pregnancy. However, the contrast agents injected for MRI imaging (category $\mathrm{C}$ of the US Food and Drug Administration drug-risk classification system) can pass through the placenta and be secreted into the amniotic fluid by the fetus's kidneys. The duration of the stay of these 
agents in the amniotic fluid is still unclear. Additionally, the chelating agents from the contrast agent's metabolites are toxic and may affect fetal development. Thus, contrast agents should be avoided when MRI scans are performed during pregnancy. However, MRI scans without contrast are of little assistance in diagnosing ovarian masses $(11,12)$. Additionally, a long duration in the supine position during late pregnancy may cause a risk of supine hypotension in pregnant women. As CT and MRI have limited application during pregnancy, dynamic observation via ultrasound is an essential tool for diagnosing ovarian masses in pregnancy and for differential diagnosis. Ultrasonic features have an important reference value in determining the nature of a mass. As this study showed, ultrasound examinations are very important in determining the nature of a mass.

\section{Clinical indications for pathological features of ovarian masses in pregnancy}

The results of this study showed an extensive source of pathological tissue for persistent adnexal masses in pregnancy. The proportion of metastatic ovarian tumors, which endanger patients' lives, was higher in pregnant women than non-pregnant women. Thus, malignant tumors in pregnancy led to a poorer prognosis. The results showed that the proportion of borderline tumors in primary ovarian tumors in pregnancy was higher than those in nonpregnancy; thus, primary ovarian masses in pregnancy led to a better prognosis. Determining the source of newly found tumors in pregnancy is very important. Metastatic tumors require an active exploratory laparotomy. However, there is value in delaying treatment to allow fetus survival, as more borderline tumors in primary ovarian tumors exist. Both tumor and fetal factors need to be taken into consideration when deciding treatment. Tumor factors include pathology, and complications. Thus, an active surgical exploration is the best choice for abdominal emergencies, suspected mass pedicle torsion, and suspected borderline/malignant tumors.

The limitation of the study is that it is not the whole population, but only the data of one center. Among the patients with persistent adnexal masses, 43 patients had persistent masses during pregnancy and postpartum, but refused surgical treatment. Therefore, the composition of pathological types may be biased.

In conclusion, this study showed that the histopathological compositions of adnexal masses in pregnancy differed to those in non-pregnancy. Further, a very high proportion of ovarian functional cysts was observed. Elective surgery during pregnancy is safe. Deciding on a treatment option requires the consideration of a number of features, the possible complications of the adnexal mass in pregnancy, and the nature of the mass.

\section{Acknowledgments}

Funding: The present study was supported by the Key Projects of Sailing Plan of Beijing Medical Administration (No. ZYLX201713).

\section{Footnote}

Reporting Checklist: The authors have completed the STROBE reporting checklist. Available at http://dx.doi. org/10.21037/atm-21-2137

Data Sharing Statement: Available at http://dx.doi. org/10.21037/atm-21-2137

Conflicts of Interest: All authors have completed the ICMJE uniform disclosure form (available at http://dx.doi. org/10.21037/atm-21-2137). The authors have no conflicts of interest to declare.

Ethical Statement: The authors are accountable for all aspects of the work in ensuring that questions related to the accuracy or integrity of any part of the work are appropriately investigated and resolved. The clinical study was approved by the Ethic Committee of Beijing ChaoYang Hospital (No. 2021-science-210) and was conducted in accordance with the provisions of the Declaration of Helsinki (as revised in 2013). Individual consent for this retrospective analysis was waived.

Open Access Statement: This is an Open Access article distributed in accordance with the Creative Commons Attribution-NonCommercial-NoDerivs 4.0 International License (CC BY-NC-ND 4.0), which permits the noncommercial replication and distribution of the article with the strict proviso that no changes or edits are made and the original work is properly cited (including links to both the formal publication through the relevant DOI and the license). See: https://creativecommons.org/licenses/by-nc-nd/4.0/.

\section{References}

1. Hoover K, Jenkins TR. Evaluation and management 
of adnexal mass in pregnancy. Am J Obstet Gynecol 2011;205:97-102.

2. Oprescu ND, Ionescu CA, Drăgan I, et al. Adnexal masses in pregnancy: perinatal impact. Rom J Morphol Embryol 2018;59:153-8.

3. Türkçüoğlu I, Meydanli MM, Engin-Ustün Y, et al. Evaluation of histopathological features and pregnancy outcomes of pregnancy associated adnexal masses. J Obstet Gynaecol 2009;29:107-9.

4. Y acobozzi M, Nguyen D, Rakita D. Adnexal masses in pregnancy. Semin Ultrasound CT MR 2012;33:55-64.

5. Usui R, Minakami H, Kosuge S, et al. A retrospective survey of clinical, pathologic, and prognostic features of adnexal masses operated on during pregnancy. J Obstet Gynaecol Res 2000;26:89-93.

6. Conte AB, Nyingone S, Jayi S, et al. Management of adnexal masses' torsion during pregnancy. Pan Afr Med J 2020;37:17.

7. Yu C, Wang J, Lu W, et al. Analysis of adnexal mass

Cite this article as: Zhang Z, Zheng X, Zhang M, Li J, Zhao J, Zheng J, Wang S. Pathological features of persistent adnexal masses in pregnancy. Ann Transl Med 2021;9(12):973. doi: 10.21037/atm-21-2137 managed during cesarean section. Adv Clin Exp Med 2019;28:447-52.

8. Malek-mellouli M, Taamallah N, Ben Amara F, et al. Laparoscopic management of ovarian masses during pregnancy. Tunis Med 2013;91:534-8.

9. Telli E, Yalcin OT, Ozalp SS, et al. Surgical intervention for adnexal masses during pregnancy. BMJ Case Rep 2013;2013:bcr2013010324.

10. Leiserowitz GS, Xing G, Cress R, et al. Adnexal masses in pregnancy: how often are they malignant? Gynecol Oncol 2006;101:315-21.

11. Canavan TP. Sonographic Tips for Evaluation of Adnexal Masses in Pregnancy. Clin Obstet Gynecol 2017;60:575-85.

12. Biggs WS, Marks ST. Diagnosis and Management of Adnexal Masses. Am Fam Physician 2016;93:676-81.

(English Language Editor: L. Huleatt) 